\title{
The impact of the superior cavo-atrial incision in children and adults.
}

\author{
Lamia Ait-Ali ${ }^{1}$, antonio ravaglioli ${ }^{1}$, Pierluigi Festa ${ }^{1}$, Alessandro Tamburrini ${ }^{1}$, Chiara \\ Marrone $^{1}$, Magdalena Cuman ${ }^{1}$, Cecilia Farnetani ${ }^{2}$, Vitali Pak ${ }^{1}$, Mario Chiavarelli ${ }^{2}$, and \\ Duccio Federici ${ }^{1}$ \\ ${ }^{1}$ Fondazione Toscana Gabriele Monasterio per la Ricerca Medica e di Sanità Pubblica \\ ${ }^{2}$ Azienda Ospedaliera Universitaria Senese
}

July 28, 2020

\begin{abstract}
Background and aim of the study The single- and double-patch repairs are the most commonly used techniques for the surgical management of partial anomalous pulmonary venous connection associated with sinus venosus atrial septal defect. The aim of this study was to retrospectively compare early and long-term surgical outcomes in pediatric and adult patients, focusing in particular on the occurrence of postoperative rhythm disturbances. Methods 115 patients (male: 63, 54.8\%) underwent surgical repair for partial anomalous pulmonary venous connection with sinus venosus atrial septal defect. 80 patients $(70.4 \%)$ underwent surgical repair in pediatric age (<16 years old), while 35 (29.6\%) patients were operated in adulthood. 49 patients $(42.6 \%)$ underwent single-patch repair and 65 patients $(56.5 \%)$ underwent double-patch repair. In only one patient the Warden procedure was performed (0,9\%). Median follow-up time was 52 months (IQ 15.1-113). Results The type of surgical technique didn't affect the overall incidence of all rhythm disturbances ( $41 \%$ in single patch group and $35 \%$ in double patch groups, $\mathrm{p}=0.5)$. At long-term follow-up, ectopic atrial rhythm, as expression of sinoatrial node disturbance, was however significantly more frequent in the pediatric population ( $32 \%$ pediatric group and $8 \%$ adult group, $\mathrm{p}=0.02$ ). Conclusions The higher incidence of ectopic atrial rhythm in children is probably related to the closer relationship of the sinus node to the superior cavo-atrial incision, which makes iatrogenic traumatism more likely to occur. Surgical techniques that avoid any manipulation on the superior cavo-atrial junction should therefore be preferred for children undergoing partial anomalous pulmonary venous connection repair.
\end{abstract}

The impact of the superior cavo-atrial incision in children and adults.

Lamia Ait-Ali ${ }^{1} \mathrm{MD}, \mathrm{PhD}$, Antonio Ravaglioli ${ }^{1} \mathrm{MD}$, Pierluigi Festa ${ }^{1}$ MD, Alessandro Tamburrini ${ }^{1}$ MD, Chiara Marrone ${ }^{1} \mathrm{MD}$, Magdalena Cuman ${ }^{1} \mathrm{MD}$, Cecilia Farnetani ${ }^{2} \mathrm{MD}$, Vitali Pak $^{1} \mathrm{MD}$, Mario Chiavarelli ${ }^{2}$ $\mathrm{MD}, \mathrm{PhD}$ and Duccio Federici ${ }^{1} \mathrm{MD}$.

Lamia Ait Ali and Antonio Ravaglioli contributed equally to this work.

${ }^{1}$ Division of Pediatric Cardiac Surgery and Cardiology and Institute of Clinical Physiology, Monasterio Foundation, Massa, Italy.

2 Division of Cardiac Surgery, Azienda Ospedaliera Universitaria Senese, Siena, Italy.

Short running title: Impact of cavo-atrial incision

Antonio Ravaglioli

Division of Pediatric Cardiac Surgery,

Monasterio Foundation, 
Via Aurelia Sud, 54100, Massa, Italy.

Tel: +39 3713921328

Email:antonioravaglioli@gmail.com

Acknowledgement: none declared.

Funding: none declared.

Conflict of interest : none declared.

Abstract

Background and aim of the study

The single- and double-patch repairs are the most commonly used techniques for the surgical management of partial anomalous pulmonary venous connection associated with sinus venosus atrial septal defect. The aim of this study was to retrospectively compare early and long-term surgical outcomes in pediatric and adult patients, focusing in particular on the occurrence of postoperative rhythm disturbances.

\section{Methods}

115 patients (male: 63, 54.8\%) underwent surgical repair for partial anomalous pulmonary venous connection with sinus venosus atrial septal defect. 80 patients $(70.4 \%)$ underwent surgical repair in pediatric age $(<16$ years old), while 35 (29.6\%) patients were operated in adulthood. 49 patients (42.6\%) underwent single-patch repair and 65 patients $(56.5 \%)$ underwent double-patch repair. In only one patient the Warden procedure was performed (0,9\%). Median follow-up time was 52 months (IQ 15.1-113).

\section{Results}

The type of surgical technique didn't affect the overall incidence of all rhythm disturbances ( $41 \%$ in single patch group and $35 \%$ in double patch groups, $\mathrm{p}=0.5$ ). At long-term follow-up, ectopic atrial rhythm, as expression of sinoatrial node disturbance, was however significantly more frequent in the pediatric population ( $32 \%$ pediatric group and $8 \%$ adult group, $\mathrm{p}=0.02)$.

\section{Conclusions}

The higher incidence of ectopic atrial rhythm in children is probably related to the closer relationship of the sinus node to the superior cavo-atrial incision, which makes iatrogenic traumatism more likely to occur. Surgical techniques that avoid any manipulation on the superior cavo-atrial junction should therefore be preferred for children undergoing partial anomalous pulmonary venous connection repair.

\section{Introduction}

Partial anomalous venous connection (PAPVC) is defined as the abnormal venous connection between one or more pulmonary veins (PVs) and the right atrium or its tributaries, such as the venae cavae, the innominate vein or the coronary sinus (1). It accounts for approximately $3 \%$ of all congenital heart diseases (2). Anomalous right-sided PV drainage is by far the most common form of PAPVC, representing at least $90 \%$ of the cases. In these patients, the right upper and middle PVs are the most frequently involved, and they usually drain into the right atrium at the level of its lateral free wall, into the superior vena cava (SVC) or at the cavo-atrial junction (3). Left-sided PAPVC is less frequent (10\%), and it features the drainage of one or more left PVs, directly or by means of an additional vertical vein, into the innominate vein, the coronary sinus or a persistent left SVC (4).

In $90 \%$ of cases, right-sided PAPVC is associated with a sinus venosus type atrial septal defect (ASD), in most instances located near the superior cavo-atrial junction (5). An intact interatrial septum is observed in only $3 \%$ of patients with PAPVC. 
Over the past decades, several surgical strategies for the correction of the PAPVC have been described. The "single-patch" technique is utilized when the right PVs drain into the cavo-atrial junction in close proximity to the ASD. In these cases, a single patch is indeed sufficient to perform both the ASD closure and the PVs rerouting to the left atrium (6). The "double-patch" technique is generally chosen instead when the PVs are connected to the SVC above the cavo-atrial junction. In these instances, after the ASD closure and the PVs rerouting, an additional pericardial patch is used to enlarge the cavo-atrial junction, thus preventing SVC stenosis (7). The Warden procedure, originally described in 1984 (8), is a useful technique that can be performed when the PVs connection lies above the superior cavo-atrial junction. The SVC is transected just above the most superior PV and the distal caval stump is used as a channel for rerouting the pulmonary venous return into the left atrium through the ASD. Finally, the proximal stump of the transected SVC is anastomosed to the right atrial appendage (9).

The "double-decker" procedure, albeit more challenging and less widely performed, certainly deserves to be mentioned as well. It encompasses the same surgical principles of the Warden procedure, to reroute the pulmonary blood flow and to avoid any surgical manipulation of the sinoatrial (SA) node, but without division and subsequent reconnection of the SVC (10).

The most common postoperative complications after PAPVC repair are SVC stenosis, PVs stenosis and atrial arrhythmias. The latter are mostly associated to surgical procedures that entail an extensive incision of the cavo-atrial junction, as in this instance the loss of sinus rhythm with transient or permanent junctional or atrial ectopic escape is frequently observed (11). The Warden procedure and the double-decker technique avoid surgical incisions near the SA node, presenting therefore a very low incidence of atrial rhythm disturbances $(9 ; 10)$. When SVC reconstruction is performed, as in the Warden procedure and in the double-patch technique, the incidence of SVC stenosis is higher $(7 ; 9)$.

We herein present a retrospective analysis of our experience in surgical management of PAPVC associated with sinus venosus type ASD. The aim of this study was to retrospectively compare early and long-term outcomes of pediatric and adult patients in relation to different surgical techniques utilized, focusing in particular on the occurrence of postoperative rhythm disturbances.

\section{Materials and methods}

This was a single-centre, retrospective study approved by the Institutional Review Board of our hospital. All patients who underwent surgical repair of PAPVC associated with sinus venosus ASD between 1990 and 2019 were identified. Patients presenting with PAPVC and no sinus venosus ASD or with other associated significant congenital heart anomalies were excluded from the study, whereas patients with associated minor abnormalities such as persistent left SVC or bicuspid aortic valve were included. Surgical procedures and all reported postoperative complications were reviewed, while follow-up data were obtained from hospital records. The reports of the last echocardiogram performed were evaluated to identify any residual shunt or SVC obstruction. Electrocardiograms (ECG) including Holter ECG were reviewed to detect rhythm disturbances, which were classified as follows: sinus nodal dysfunction (SND), defined as "persistent sinus bradycardia inappropriate to hemodynamic requests", frequently in the form of brady-tachy syndrome (16); ectopic atrial rhythm (EAR) likely caused by iatrogenic SA node injury; atrio-ventricular (AV) block, ventricular arrhythmias and atrial fibrillation (AF). According to the timing of their occurrence, rhythm disturbances were divided in early-onset and late-onset, as to whether they developed within 30 days after surgery or over 30 days post-surgery, respectively.

Surgical repair

All procedures were carried out through a median sternotomy, under moderate hypothermic cardiopulmonary bypass (CPB). Cold blood antegrade cardioplegia was used in all cases. Glutaraldehyde-treated autologous pericardial patches were employed to perform ASD closure, PVs rerouting and cavo-atrial junction enlargement, when required. In a single case of Warden procedure, a direct SVC-right atrial appendage anastomosis was performed to correct the upper venous drainage. The surgical technique for the PAPVC repair was chosen according to the site of abnormal drainage of the PVs. When the connection was at the level of the 
superior cavo-atrial junction close to the ASD, a single-patch repair was performed. Conversely, when the anomalous PVs drained into the SVC, well above the cavo-atrial junction, a double-patch repair was preferred in nearly all cases, except from a single Warden procedure which was performed in view of surgeon's personal preference. In all cases, surgical access to the right atrium was obtained through a longitudinal atriotomy parallel to the atrioventricular groove, or through an L-shaped atriotomy. In patients where an extensive superior cavo-atrial enlargement was required (double-patch group), the incision was carried out as close as possible to the site of PVs connection to the SVC, in order to limit iatrogenic damage to the SA node. When single-patch technique was adopted instead, a more limited superior cavo-atrial incision was performed.

Data analysis

Continuous variables are expressed as mean \pm standard deviations or as median and interquartile ranges, while categorical variables are expressed as absolute numbers and percentages. Analysis of continuous variables was performed by analysis of variance (ANOVA) test or Mann Whitney test, as appropriate. The Pearson's chi-square test and the t-test were used to analyze categorical variables.

A p value $<0.05$ was considered statistically significant.

\section{Results}

Population study and early post-operative outcome.

Between October 1990 and December 2019, 115 patients (male: 63, 54.8\%), underwent surgical repair of PAPVC associated with sinus venosus ASD. The median age at the time of surgery was 8.8 years (IQ 4.323.6) (Table 1). In 80 cases $(70.4 \%)$ the repair was performed in pediatric age ( $<16$ years old), while the remaining $35(29.6 \%)$ patients were operated in adulthood (Figure 1). Surgical technique entailed singlepatch repair in 49 patients $(42.6 \%)$, double-patch repair in 65 patients $(56.5 \%)$ and one Warden procedure $(0.9 \%)$ (Table 1). No perioperative mortality was observed.

For 84 patients ( 57 in the pediatric group and 28 in the adult group), complete data regarding their postoperative period were available. Overall, the mean post-operative length of stay was $8.5 \pm 2.8$ days.

Hemodynamically significant early-onset arrhythmias were reported in 6 patients, namely one patient suffered from supraventricular tachycardia, 1 had brady-tachy syndrome requiring implantation of a pacemaker and 4 had AF. In addition, 15 patients presented early-onset EAR without hemodynamic consequences, and this was by far the commonest postoperative arrhythmia.

Early post-operative course according to age.

Post-operative complications were not different between the pediatric and the adult groups patient (Table 1). Nonetheless, in the adult group the rate of early-onset arrhythmias with hemodynamic compromise was significantly higher compared to the pediatric group, $17.8 \%$ vs $1.7 \%$, respectively $(\mathrm{p}=0.006)$. Four adult patients indeed experienced AF and another one had SND which required permanent pacemaker. The percentage of EAR was certainly increased among pediatric patients compared to the adults (21.4\% vs $10.7 \%$ ), albeit without reaching statistical significance $(\mathrm{p}=0.2)$ (Table 1$)$.

Early postoperative course according to surgical repair.

When comparing patients who underwent single-patch repair to patients who underwent double-patch repair, we did not find any statistically significant difference in terms of age at surgical repair (Table 2). Early postoperative results were also similar in these 2 groups. In particular, the early postoperative incidence of EAR was not different between the single patch group and the double patch. Even when taking into account only the pediatric population, this difference was still not significant.

Follow-up outcome ( $>30$ days). 
Forty-five patients were lost at follow-up, thus 70 patients (23 adults and 47 pediatrics) were enrolled in the subsequent statistical analysis. There was no statistical difference between patients lost at follow-up and the remaining population in terms of gender, age and type of surgical repair (supplementary table 1).

Median follow-up time was 52 months (15.1-113) and median age at follow-up was 16 years (10.5- 35.7). SVC obstruction was detected at echocardiogram and confirmed with cardiac magnetic resonance imaging in 2 pediatric patients both operated with the double-patch technique, although additional surgical or interventional procedure was required in these cases. We didn't observe any mortality in our follow-up group.

At their last follow-up 58 patients $(82.9 \%)$ were in sinus rhythm, 41 in the pediatric group and 17 in the adult group $(\mathrm{p}=0.1)$. The other 12 patients $(17.1 \%)$ instead had developed late-onset rhythm disturbances. Five children had non-hemodynamically significant EAR. Among the adults, 2 had SND with subsequent pacemaker implantation, 2 had frequent premature ventricular contractions requiring antiarrhythmic drugs, another 2 had AF and finally 1 adult patient had second degree AV block. Significant statistical difference $(\mathrm{p}=0.04)$ was therefore observed in terms of types of late-onset rhythm disturbance between the pediatric patients and the adult patients (Table 3). Nonetheless, in regard to the type of surgical repair, no statistical difference $(\mathrm{p}=0.2)$ in occurrence of late-onset arrhythmias was highlighted when comparing the single-patch $(24.1 \%)$ and the double-patch group (12.5\%).

Cumulative rhythm anomalies analysis.

We finally evaluated the overall occurrence of any rhythm disturbance among operated patients, considering both early-onset arrhythmias and late-onset arrhythmias (Figure 2). A total of 29 (33,3\%) patients had experienced at least one arrhythmia, either in the early postoperative period or at follow-up (Table 4).

Surgical technique alone did not seem to affect the overall occurrence of all rhythm disturbances $(41 \%$ in single patch group and $35 \%$ in double patch groups, $\mathrm{p}=0.5)$ and of EAR alone ( $29 \%$ in single patch group and $24.4 \%$ in double patch group, $\mathrm{p}=0.4)$. On the other hand, cumulative onset of EAR was significantly more frequent $(\mathrm{p}=0.02)$ among the pediatric patients compared to the adults, $32 \%$ vs $8 \%$, respectively. Finally, as possibly expected from the separate early-onset and late-onset analysis, we observed that overall most of the arrhythmias with hemodynamic compromise occurred in the adult population, and this result was statistically significant $(\mathrm{p}=0.006)$ (Table 1$)$.

\section{Conclusions}

In this retrospective study we compared the early, late and overall occurrence of arrhythmias in adult and pediatric patients who underwent surgical repair of PAPVC + sinus venosus ASD over a thirty-years period. Nearly all patients were treated by means of single-patch and double-patch surgical technique ( $42.6 \%$ and $56.5 \%$ respectively). The incidence of transient and permanent arrhythmias in relation to the age at repair and to the type of surgical procedure is the main endpoint of our analysis. When comparing the surgical techniques, we did not find a significant difference between the 2 groups in regard to overall incidence rate of arrhythmias at any point of follow-up. In particular, the presence of EAR, intended as a direct consequence of an iatrogenic injury to the SA node, was not different between double- and single-patch repair groups. This is consistent with the work of Said et al (12), who did not observe a significant difference in the incidence number of SND and supraventricular arrhythmias among 124 patients treated by single or double patch technique or Warden procedure. On the contrary, other institutions reported different results in terms of incidence of postoperative arrhythmias between surgical groups, with a significantly higher frequency of non-sinus rhythm in patients who underwent double-patch repair. Stewart et al (13) showed a 55\% incidence of supraventricular arrhythmias in the double-patch group, compared to significant lower incidence in singlepatch and Warden groups (24\% and 0\%, respectively). Similarly, a recent paper by Jaschinski et al (14) displayed analogous features, where patients treated with the double-patch technique had a significantly higher incidence of non-sinus rhythm.

The reason behind the higher risk of iatrogenic SA node injury using the double-patch repair technique lies intuitively in the need for a more extensive superior cavo-atrial incision, which is a peculiar feature of this 
operation (7). In this surgical scenario, the possibility of damaging the SA node is certainly increased than in other procedures where the surgeon remains far away from this area, such as in the Warden and in the double-decker operations $(10 ; 15)$.

The SA node is the natural pacemaker of the heart. It is a complex sub-epicardial structure located in the antero-lateral aspect of the superior cavo-atrial junction. Its yellowish appearance on the external surface should allow the surgeon to identify and avoid it when carrying out the cavo-atrial incision. Nevertheless, anatomic variations of its extension and blood supply are common, thus explaining the high rate of postoperative SA node dysfunction.

In our analysis, we found a significantly higher incidence of SA node impairment in the pediatric population, and we acknowledge this may be of great importance. Perioperative incidence (early-onset) of EAR had a non-statistically significant increased trend in children compared to the adults, but when considering the longterm follow-up this difference becomes statistically significant, as EAR occurred overall in $32 \%$ of pediatric cases vs $8 \%$ of adult cases $(\mathrm{p}=0.02)$. In our opinion, this is likely to be the result of a much more common iatrogenic injury of the SA node in smaller patients, no matter which surgical technique is utilized. In young children indeed, the presence of small anatomical structures forces the surgeon to perform the cavo-atrial incision very close to the conduction system, increasing the risk of damaging the SA node. In adults instead, restoration of sinus rhythm over time occurred more frequently, perhaps because the larger dimension of the superior cavo-atrial junction and its distance from the SA node, makes the incision of cavo-atrial junction less likely to affect the conduction system. Conversely though, arrhythmias causing hemodynamic consequences were a significantly more common complication in adult patients, both in the early post-operative period and long-term. This is probably due, at least in part, to the long-standing volume overload and to the consequent distention of the right atrial fibres.

To our knowledge, this is the first study to analyze the different timing and pattern of post-operative arrhythmias in pediatric and adult patients undergoing PAPVC + sinus venosus ASD repair. We strongly believe that the significantly higher incidence of permanent SND with junctional or atrial ectopic escape among the pediatric patients could be related to the limited space between SA node area and cavo-atrial incision. In single-patch and especially in double-patch technique, a surgical incision of variable extension is performed across the cavo-atrial junction very close to the SA node (Figure 3). When the distance between the PV connection plan and the SA node is relatively wide, as in adult patients, the chance to interfere with the SA node activity is fairly minimal. On the other hand, when this distance is shorter, such as in pediatric patients, the probability of SA node dysfunction is increased. Therefore, the choice of surgical technique may play a crucial role to avoid the occurrence of iatrogenic SA node injuries causing certain arrhythmias, especially in the pediatric population. As initially mentioned, the Warden procedure is the most reproducible option to avoid surgical damage to the SA node, and there is nowadays increasing evidence of its superiority in terms of postoperative freedom from SND and EAR, compared to single- and double-patch procedures $(9 ; 10)$.

If results are confirmed by further larger studies, surgical procedures that completely avoid invasiveness and trauma at the level of superior cavo-atrial junction should probably be preferred in pediatric patients. Among the available techniques, although not free from other complications, the Warden procedure could be considered the safest and most reproducible (15). In our study population only one patient underwent the Warden procedure, and this was due exclusively to the surgeon's preference. In view of our results and new insights, we believe that multicenter randomized prospective studies are needed to compare the arrhythmogenic effects of the Warden and double-patch techniques in children. In adult patients, in whom Warden procedure may present technical challenges in view of a lower mobility of the SVC, the doublepatch technique will remain our preferred approach. Further studies are needed to specifically investigate the impact of EAR during growth in the pediatric population and to validate the protocol that will currently advocate at our institution.

The retrospective nature and the relatively small sample size are the main limitations of the present study. The bias may potentially be less if we take into account the consistency of a single center and the homogeneous 
surgical strategy, nevertheless larger and prospective studies are certainly warranted.

We also acknowledge that the follow-up was not complete for all patients, as 45 of them were lost at follow-up and were therefore excluded from the analysis. Nonetheless, their demographic and surgical characteristics were not dissimilar to those of patients who completed the follow-up.

The surgical management of PAPVC with sinus venosus ASD, requiring superior cavo-atrial incision, is associated with high incidence of postoperative SND (16). In our experience, this finding has been significantly higher among pediatric patients, probably due to the smaller cavo-atrial junction size and the subsequent close proximity of the SA node to the PV connection site and surgical incision, hence increasing the risk of iatrogenic damage. Surgical techniques which avoid manipulation of the superior cavo-atrial junction should therefore be preferred in pediatric patients.

\section{Figure legends:}

Figure 1. Bar graph showing the age distribution at the time of surgical repair.

Figure 2. Bar graph showing the overall arrhythmias in the two age-groups.

Figure 3. Relationship between the surgical incision and the SA node.

Table 1. Surgical repair and postoperative complications according to the age-group.

\begin{tabular}{|c|c|c|c|c|}
\hline & Total (115) & Age $<16$ years $(80)$ & $(35)$ & $p$ \\
\hline $\begin{array}{l}\text { Gender male, n. } \\
(\%)\end{array}$ & $63(54.8)$ & $46(56.8)$ & $17(50)$ & 0.3 \\
\hline $\begin{array}{l}\text { Age at complete } \\
\text { repair, years (IQ) }\end{array}$ & $8.84(4.3-23.6)$ & $5.9(3.3-9.4)$ & $41.5(29.3-52.8)$ & $<0.001$ \\
\hline $\begin{array}{l}\text { Type of repair, n. } \\
(\%) \text { Single Patch } \\
\text { Double patch } \\
\text { Warden }\end{array}$ & $\begin{array}{l}49(42.6) 65(56.5) 1 \\
(0.9)\end{array}$ & $\begin{array}{l}33(40.7) 47(58) 1 \\
(1.3)\end{array}$ & $16(47.1) 18(52.9) 0$ & 0.6 \\
\hline $\begin{array}{l}\text { LOS hospital, } \\
\text { days }\end{array}$ & $8.5 \pm 2.8$ & $8.3 \pm 2.9$ & $8.8 \pm 2.6$ & 0.4 \\
\hline $\begin{array}{l}\text { Post. op. } \\
\text { outcome n. } \\
\text { patients (\%) }\end{array}$ & $\begin{array}{l}849(10.5) 2(2.3) \\
2(2.3) 1(1.1) 6 \\
(5.8) 5115(17.6)\end{array}$ & $\begin{array}{l}567(12.5) \quad 1111 \\
(1.7) 1012(21.4)\end{array}$ & 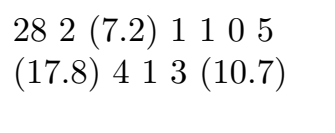 & $0.4 \mathbf{0 . 0 0 6} 0.2$ \\
\hline \multicolumn{5}{|l|}{$\begin{array}{l}\text { Pericardial } \\
\text { effusion }\end{array}$} \\
\hline \multicolumn{5}{|l|}{ Pneumothorax } \\
\hline \multicolumn{5}{|l|}{$\begin{array}{l}\text { Neurologic } \\
\text { complications }\end{array}$} \\
\hline \multicolumn{5}{|l|}{ Ventricular } \\
\hline \multicolumn{5}{|l|}{ Symptomatic } \\
\hline \multicolumn{5}{|l|}{ Atrial } \\
\hline \multicolumn{5}{|l|}{ fibrillation/SVT } \\
\hline \multicolumn{5}{|l|}{ SND } \\
\hline EAR & & & & \\
\hline
\end{tabular}

EAR: ectopic atrial rhythm; LOS: length of stay; n.: number; SND: sinus node dysfunction, SVT: supraventricular. 
Table 2. Surgical repair and postoperative complications according to the surgical technique.

\begin{tabular}{|c|c|c|c|c|}
\hline & Total (115) & Single patch (49) & Double patch (65) & $p$ \\
\hline $\begin{array}{l}\text { Gender male, } \mathrm{n} . \\
(\%)\end{array}$ & $63(54.8)$ & $28(57.1)$ & $34(52.3)$ & 0.3 \\
\hline $\begin{array}{l}\text { Age at complete } \\
\text { repair, years (IQ) }\end{array}$ & $8.84(4.3-23.6)$ & $8.8(4-27.8)$ & $8.9(4.6-20)$ & 0.9 \\
\hline $\begin{array}{l}\text { LOS hospital, } \\
\text { days }\end{array}$ & $8.5 \pm 2.8$ & $8 \pm 2.1$ & $8.8 \pm 3.2$ & 0.19 \\
\hline $\begin{array}{l}\text { Post. op. } \\
\text { outcome, n. (\%) }\end{array}$ & $\begin{array}{l}859(10.5) 2(2.3) \\
2(2.3) 1(1.1) 6\end{array}$ & $\begin{array}{l}262^{*}(7.6) 1102 \\
(5.5) 117(26.9)\end{array}$ & $\begin{array}{l}377(18.9) 1114 \\
(10.8) 408(21.6)\end{array}$ & $\begin{array}{lll}0.1 & 0.6 & 0.8\end{array}$ \\
\hline Pericardial & $(5.8) 5115(19)$ & & & \\
\hline \multicolumn{5}{|l|}{ Effusion } \\
\hline \multicolumn{5}{|l|}{ Pneumothorax } \\
\hline \multicolumn{5}{|l|}{$\begin{array}{l}\text { Neurologic } \\
\text { complications }\end{array}$} \\
\hline \multicolumn{5}{|l|}{ Ventricular } \\
\hline \multicolumn{5}{|l|}{ dysfunction } \\
\hline \multirow{2}{*}{\multicolumn{5}{|c|}{$\begin{array}{l}\text { Symptomatic } \\
\text { arrhythmias }\end{array}$}} \\
\hline & & & & \\
\hline \multicolumn{5}{|l|}{ Atrial fibrillation } \\
\hline \multicolumn{5}{|l|}{ SND } \\
\hline EAR & & & & \\
\hline
\end{tabular}

EAR: ectopic atrial rhythm; LOS: length of stay, n.: number; SND: sinus node dysfunction, SVT: supraventricular.

*One patient experienced atrial arrhythmias and pericardial effusion.

Table 3. Rhythm anomalies at follow-up according to the age-group.

\begin{tabular}{lllll}
\hline & Total $(70)$ & Age $<16$ years $(47)$ & $(23)$ & $p$ \\
\hline $\begin{array}{l}\text { Follow-up time, } \\
\text { months (IQ) }\end{array}$ & $52(15.1-113)$ & $54(22-123.6)$ & $39.3(13-87.2)$ & 0.5 \\
Sinus rhythm (\%) & $58(82.9) 5(7.1) 2$ & $41(87.2) 5(10,6)$ & $17(73.9) 02(8.7)$ & $0.10 .1 \mathbf{0 . 0 4} \mathbf{0 . 0 4}$ \\
EAR (\%) SND & $(2.9) 2(2.9) 2$ & 0000 & $2(8.7) 2(8.7) 1$ & $\mathbf{0 . 0 4} 0.3$ \\
$\begin{array}{l}\%) \text { Atrial } \\
\text { fibrillation and }\end{array}$ & $(2.9) 1(1.4)$ & & & \\
SVT (\%) & & & & \\
Ventricular & & & & \\
arrhythmias $(\%)$ & & & & \\
AV block (\%) & & & & \\
\hline
\end{tabular}

AV: atrio-ventricular; EAR: ectopic atrial rhythm; SND: sinus node dysfunction, SVT: supraventricular.

Table 4. Overall rhythm anomalies according to the age-group. 


\begin{tabular}{lllll}
\hline & Total $(75)$ & Age $<$ 16years $(50)$ & $(25)$ & $p$ \\
\hline Rhythm & $46(61.3) 18(24)$ & $31(62) 16(32) 0$ & $15(60) 2(8) 2(8)$ & $\mathbf{0 . 0 0 8} 0.8 \mathbf{0 . 0 2}$ \\
anomalies (\%) & $2(2.7) 6(8) 2$ & $1(2) 1(2) 1(2)$ & $5(20) 1(4) 0(0)$ & $\mathbf{0 . 0 4} \mathbf{0 . 0 0 7} 0.6$ \\
Sinus rhythm & $(2.9) 1(1.4)$ & & & 0.4 \\
EAR & & & & \\
SND & & & & \\
Atrial fibrillation & & & & \\
and SVT & & & & \\
Ventricular & & & & \\
arrhythmias & & & & \\
AV block & & & & \\
\hline
\end{tabular}

AV: atrio-ventricular; EAR: ectopic atrial rhythm; SND: sinus node dysfunction, SVT: supraventricular.

\section{Author contributions}

L.Ait-Ali: concept/design, data analysis/interpretation/collection, statistics, critical revision, approval final version.

A.Ravaglioli: concept/design, data analysis/interpretation/collection, drafting article, critical revision, approval final version.

P.Festa: data interpretation, critical revision, approval final version.

A.Tamburrini: data interpretation, critical revision, approval final version.

C.Marrone: data interpretation, critical revision, approval final version.

M.Cuman: data interpretation, critical revision, approval final version.

C.Farnetani: data acquisition, critical revision, approval final version.

V.Pak: data analysis/interpretation, critical revision, approval final version.

M.Chiavarelli: data acquisition/interpretation, critical revision, approval final version.

D.Federici: concept/design, data analysis/interpretation, critical revision, drafting article, approval final version.

\section{References}

1. DeLeon SY, Freeman JE, Ilbawi MN, Husayni TS, Quinones JA, Ow EPet al . Surgical techniques in partial anomalous pulmonary veins to the superior vena cava. Ann Thorac Surg 1993;55 (5): 1222-1226.

2. Aboukhoudir F, Aboukhoudir I, Pansieri M, Khennine B, Hirsch JL, Rekik S. Partial anomalous pulmonary venous return in a 64-year-old woman. Ann Cardiol Angiol (Paris) 2015;64 (05):394-398.

3. Kim C, Cho YH, Lee M, Yang JH, Jun TG, Song JY et al . Surgery for partial anomalous pulmonary venous connection: Modification of the Warden procedure with a right atrial appendage flap. Korean J Throrac Cardiovasc Surg 2014;47 (02):94-99.

4. Aguilar Jiménez JM, Torres EG, Arlati F, Comas J, Illas JV. Unnoticed bilateral partial pulmonary vein connection. J Thorac Cardiovasc Surg 2015:149 (03): e40-e42.

5. Szychta W, Cerin G, Popa BA, Armienti F, Lanzillo G, Diena M et al . Atypical abnormal pulmonary vein drainage with atrial septal defect: Surgical treatment. Echocardiography 2015;32(06): 1040-1043.

6. Iyer AP, Somanrema K, Pathak S, Manjiunath PY, Pradhan S, Krishman S. Comparative study of single- and double-patch techniques for sinus venous atrial septal defect with partial anomalous pulmonary venous connection. J Thorac Cardiovasc Surg 2007;133(03): 656-659. 
7. Van De Woestijne PC, Verberkmoes N, Bogers AJ. Partial anomalous pulmonary venus connection (including scimitar syndrome). Multimed Man Cardiothorac Surg 2013;2013:mmt001.

8. Kottayl BP, Dharan BS, Menon S, Bijulal S, Neema PK, Gopalakrishnan SKet al . Anomalous pulmonary venous connection to superior vena cava: Warden technique. Eur J Cardiothorac Surg 2011;39(03): 388-391.

9. Gustafson RA. Cavo-atrial anastomosis technique for partial anomalous pulmonary venous connection to the superior vena cava: The Warden procedure. Oper Tech Thorac Cardiovasc Surg 2006;11(01):2232 .

10. Hongu H, Yamagishi M, Maeda Y, Itatani K, Asada S, Fujita S et al . Double-decker repair for partial anomalous pulmonary venous return into the superior vena cava. J Thorac cardiovasc Surg 2019 May; 157(5):1970-1977.

11. Okonta KE, Agarwal V. Does Warden procedure reduce sinus node dysfunction after surgery for partial anomalous pulmonary venous connection? Interact Cardiovasc Thorac Surg 2012:14(06):839-842.

12. Said SM, Burkhart HK, Schaff HV, Cetta F, Phillips SD, Barnes RD et al . Single-patch, 2-patch and caval division techniques for repair of partial anomalous pulmonary venous connection: Does it matter? J Thorac Cardiovasc Surg 2012; 143(04): 896-903.

13. Stewart RD, Baillard F, Kelle AM, Backer CL, Young L, Mavroudis C. Evolving surgical strategy for sinus venous atrial septal defect: Effect on sinus node function and late venous obstruction. Ann Thorac Surg 2007 Nov; 84(5): 1651-5.

14. Jashinski C, Cussig C, Fonseca E, Brukner T, Kark M, Loukanov T. A wide comparison of techniques for repair of PAPVC: One institution's 20-year experience. Thorac Cardiovasc Surg 2020 Jan; 68(1): $15-23$.

15. Lin H, Yan J, Wang Q, Li S, Sun H, Zhang Y et al . Outcome of the Warden procedure for partial anomalous pulmonary venous drainage. Pediatr cardiol 2020 Jan; 41(1): 134-140.

16. Buz S, Alexi-Meskishvili V, Villavivencio, Lorini F, Hubler M, Weng Y Berger F et al . Analysis of arrhythmias after correction of partial anomalous pulmonary venous connection. Ann Thorac Surg 2009 Feb; 87(2): 580-3. 


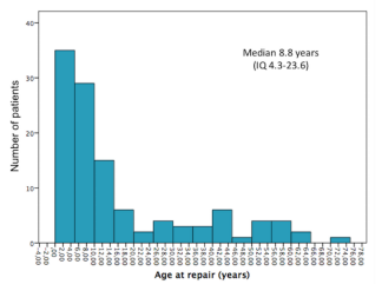




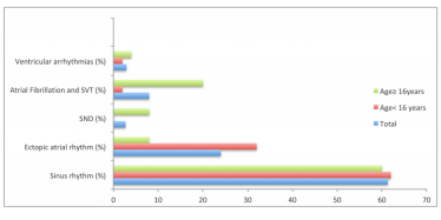




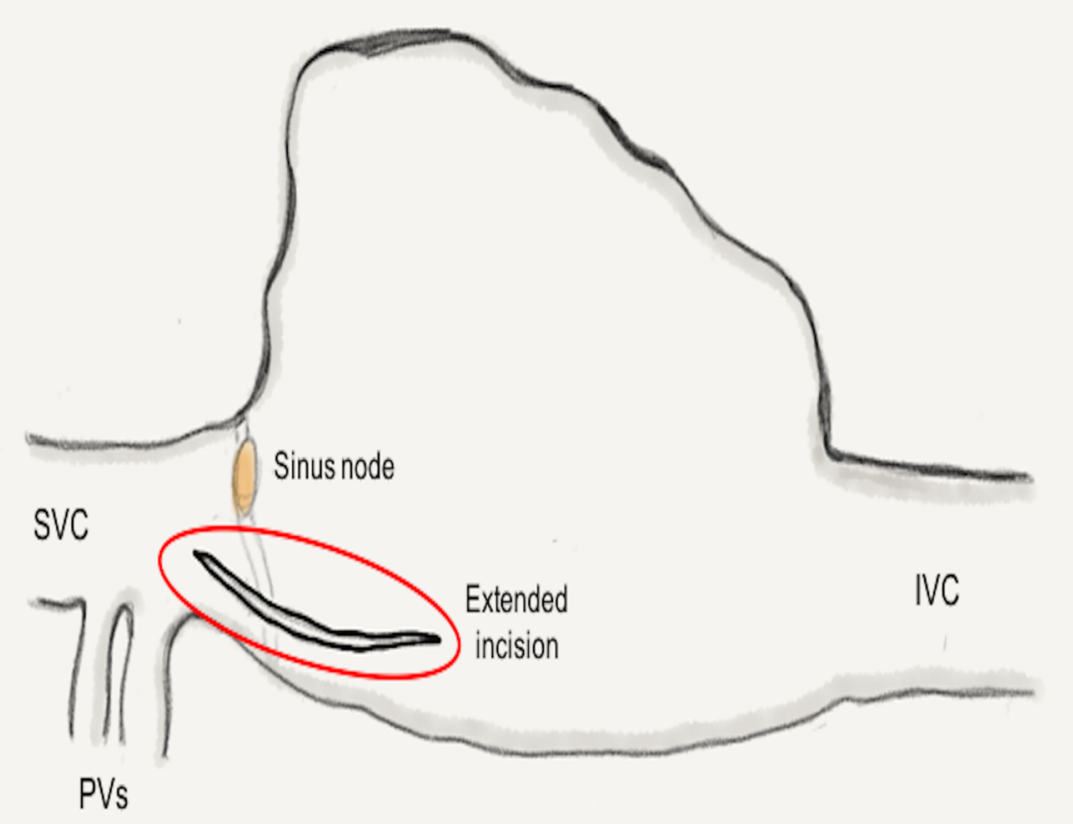

\section{Hosted file}

Table 1.docx available at https://authorea.com/users/346808/articles/472659-the-impact-ofthe-superior-cavo-atrial-incision-in-children-and-adults

\section{Hosted file}

Table 2.docx available at https://authorea.com/users/346808/articles/472659-the-impact-ofthe-superior-cavo-atrial-incision-in-children-and-adults

\section{Hosted file}

Table 3.docx available at https://authorea.com/users/346808/articles/472659-the-impact-ofthe-superior-cavo-atrial-incision-in-children-and-adults

\section{Hosted file}

Table 4.docx available at https://authorea.com/users/346808/articles/472659-the-impact-ofthe-superior-cavo-atrial-incision-in-children-and-adults 